\title{
Let-7d suppresses growth, metastasis, and tumor macrophage infiltration in renal cell carcinoma by targeting COL3A1 and CCL7
}

Boxing Su ${ }^{1,2+}$, Wei Zhao ${ }^{3 \dagger}$, Bentao Shi ${ }^{4}$, Zhongyuan Zhang ${ }^{1,2}, X_{i} Y^{1,2}$, Feng Xie ${ }^{1,2}$, Zhongqiang Guo ${ }^{1,2}$, Xiaoyu Zhang ${ }^{1,2}$, Jin Liu ${ }^{1,2}$, Qi Shen ${ }^{5}$, Jinghua Wang ${ }^{5}$, Xuesong Li, ${ }^{1,2}$, Zhiqian Zhang ${ }^{3^{*}}$ and Liqun Zhou ${ }^{1,2^{*}}$

\begin{abstract}
Background: MicroRNAs are endogenous small noncoding RNAs that are functionally involved in numerous critical cellular processes including tumorigenesis. Data mining using a microRNA array database suggested that let-7d microRNA may be associated with renal cell carcinoma (RCC) malignant progression. Here, we performed further analyses to determine whether let-7d is functionally linked to RCC malignancy.

Methods: Quantitative real-time PCR was used to determine the level of mature let-7d in RCC clinical specimens and its correlation with clinicopathological data. Immunohistochemical staining was conducted to characterize the stroma of RCC. Let-7d overexpressing RCC cell lines combined with mouse models bearing cell-derived xenografts and patient-derived xenografts were used to assess the functional role of let-7d in vitro and in vivo.

Results: Downregulation of let-7d in clinical RCC samples was associated with advanced tumor grade and T stage and increased vascular invasion. An inverse relationship between let-7d expression and macrophage infiltration was found in clinical RCC samples. Functional studies indicated that ectopic expression of let-7d significantly inhibited RCC cell proliferation, migration, and peripheral blood monocyte (PBMC) recruitment in vitro, as well as tumor growth, metastasis, and tumor macrophage infiltration in vivo. In silico analysis and subsequent experimental validation confirmed collagen, type III, alpha 1 (COL3A1) and C-C subfamily chemokine member CCL7 as direct let-7d target genes. The addition of COL3A1 and CCL7 counteracted the inhibitory effects of let-7d on RCC cell proliferation, migration, and PBMC recruitment. The inhibition of let-7d increased cell proliferation, migration, and PBMC recruitment by the enhanced expression of COL3A1 and CCL7 genes in vitro. The mRNA levels of COL3A1 and CCL7 were inversely correlated with let-7d level in RCC clinical specimens.
\end{abstract}

Conclusions: These results suggest that let-7d may suppress RCC growth, metastasis, and tumor macrophage infiltration at least partially through targeting COL3A1 and CCL7.

Keywords: Renal cell carcinoma, MicroRNA, Let-7

\footnotetext{
* Correspondence: zlzqzhang@bjmu.edu.cn; zhoulqmail@china.com

${ }^{\dagger}$ Equal contributors

${ }^{3}$ Department of Cell Biology, Peking University School of Oncology, Beijing

Cancer Hospital and Institute, Beijing 100142, China

'Department of Urology, Peking University First Hospital \& the Institute of Urology, Peking University, Beijing 100034, China

Full list of author information is available at the end of the article
} 


\section{Background}

Renal cell carcinoma (RCC) is one of the common urological cancers usually with poor prognosis [1]. RCC accounts for approximately 3\% of adult malignancies and for approximately 90-95\% of neoplasms arising from the kidney [2,3]. Surgical treatment can cure $60-70 \%$ of localized RCC but only prolongs survival in most metastatic RCC patients [4]. RCC is relatively resistant to radiation and chemotherapy [5]. Considerable progress has been made in the therapy of patients with localized RCC; however, the treatment options for patients with metastatic RCC are very limited [6]. Therefore, identification of new biomarker and anti-tumor agents, particularly with better efficiency against metastatic RCC, remains a high priority.

MicroRNAs (miRNAs) are a large gene family of short (21-23 nucleotides) non-coding RNAs. The single-stranded miRNA binds through imperfect base pairing with the 3' untranslated region ( $3^{\prime}$-UTR) of target mRNAs and causes either repression of translation or degradation of mRNAs [7]. Each of the hundreds of known miRNAs can epigenetically downregulate many target genes that participate in various biological processes including cell proliferation, apoptosis, migration, differentiation and development [8].

It is increasingly apparent that the interplay between cancer cells and their stroma is of great importance to tumorigenesis and progression [9]. Tumor-associated stroma is composed of multiple stromal cell types, such as cancer-associated fibroblasts, immune inflammatory cells, and endothelial cells, as well as a variety of extracellular matrix (ECM) proteins, such as fibronectin and collagen. The tumor-associated stroma constitutes an important compartment of tumor microenvironment, which can enable primary, invasive, and then metastatic growth of tumor through crosstalk with cancer cells [10]. Recent studies have shown that the regulatory role of miRNAs during cancer progression is not limited to cancer cells, and that miRNAs are also involved in the activation and transition of tumor stromal cells [11]. Thus, miRNAs have emerged as a potent regulator in the crosstalk between cancer and stromal cells in the tumor microenvironment.

The let-7 family, originally identified in Caenorhabditis elegans, consists of 13 family members that are highly conserved across the animal phylogeny from C. elegans to human. Nine members of the let- 7 family have been identified in humans [12]. Let-7 functions as a heterochronic gene in many species. It is undetectable in human and mouse embryonic stem cells, but increases during embryogenesis and differentiation [13]. High let-7 expression levels are subsequently maintained in a variety of adult tissues [14]. Conversely, let-7 is frequently downregulated in many human malignancies, such as lung cancer, breast cancer, and hepatocellular carcinoma [15-17], possibly reflecting the reverse embryogenesis process that occurs during oncogenesis [18].

Recently, we have identified a subset of miRNAs that are low expressed in RCC relative to adjacent normal tissues by using microarray (unpublished data). The current study was designed to explore the function of one of these miRNAs, let-7d, in RCC progression.

\section{Results}

Let-7d is downregulated in human RCC cell lines and clinical RCC samples

We examined let-7d expression in several human RCC cell lines by quantitative real-time RT-PCR. The normal renal tubule epithelial cell line HK-2 had significantly higher let-7d level than the RCC cell lines (Figure 1A). We then examined let-7d expression in 80 clinical RCC samples and their matched adjacent tissues. The mean let-7d level in RCC was $17.6 \%$ of that in the matched adjacent tissues (Figure 1B). Given that let-7 family members are sometimes indistinguishable [19], we also checked the expression of let-7a, the only let-7 family member that was reported to function as a tumor suppressor in RCC cell lines [20]. As shown in Additional file 1: Figure S1 (Supplementary Data), no difference of let-7a expression was found between tumor tissues and the matched adjacent tissues. These data indicate that the decrease of let-7d expression in RCC may be specific.

\section{Decreased let-7d expression is associated with advanced $\mathrm{T}$ stages and tumor grades in RCC patients}

A total of 80 RCC patients were analyzed for the correlation between clinicopathological characteristics and the let-7d level in RCC (Table 1, Figures $1 \mathrm{C}$ and 1D). There was no significant correlation between let-7d level and age at surgery or histological subtype. Let-7d level in RCC was significantly lower in male patients than in female patients (Table 1), but the difference between males and females was not found in the adjacent normal tissues (Additional file 1: Figure S2). Let-7d expression was significantly lower in T3 stage RCC than in T1 and T2 stage RCC, and decreased remarkably with advanced degree of RCC differentiation. Moreover, let-7d expression in RCC with vascular invasion was significantly lower than that in RCC without vascular invasion. Therefore, downregulation of let- $7 \mathrm{~d}$ is highly correlated with the malignant degree of RCC.

\section{Let-7d expression is inversely correlated with tumor macrophage infiltration}

We investigated the association between let-7d expression and changes of several tumor stromal cells including macrophages, cancer-associated fibroblasts, T-regulatory cells (Tregs), and mast cells, which have been well documented to associate with tumor progression [10,21,22]. 

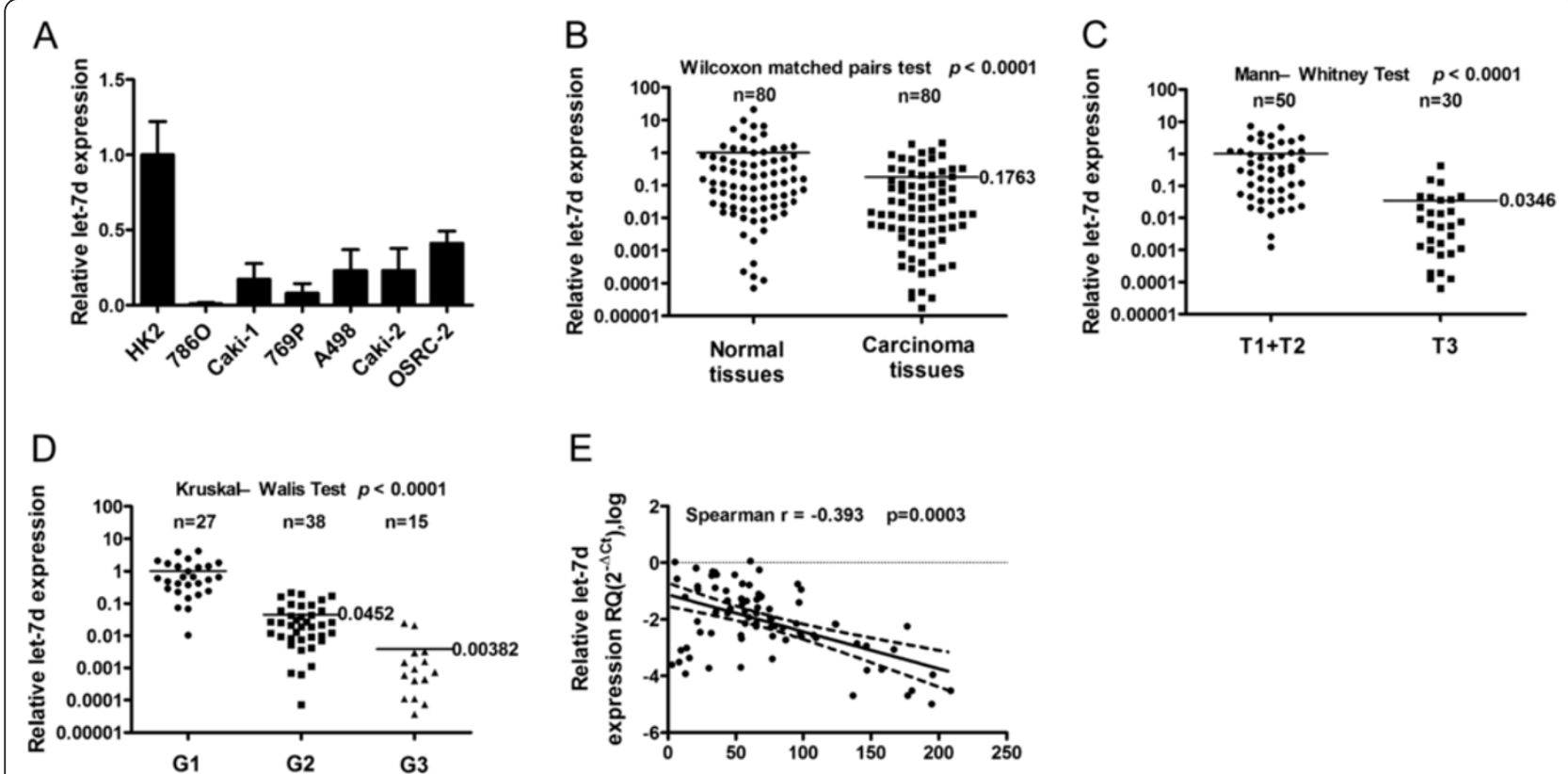

E

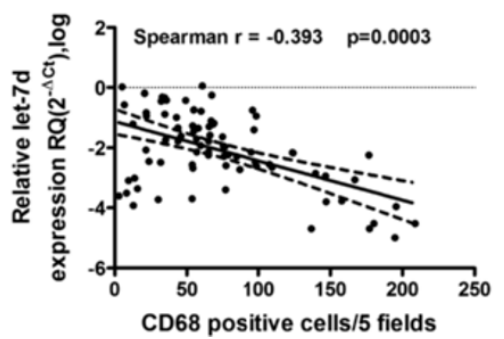

Figure 1 Let-7d expression in RCC cell line and tumor samples, and correlation with tumor stromal cells. (A) Real-time RT-PCR analysis of relative let-7d expression in human cell lines. The data represent the average \pm SD of three independent RT-PCR results. (B) The relative expression of let-7d in 80 RCC tissues and matched adjacent normal tissues were analyzed by real-time RT-PCR. (C, D) Real-time RT-PCR analysis of relative let-7d expression in 80 RCC tissues in different T stages (C) and tumor grades (D). Horizontal lines in (B-D) represent the mean values of relative let-7d expression for each series of samples. Note that (B-D) were generated from the same data as Table 1. (E) A linear regression and correlation among relative let-7d expression in a log scale vs. CD68 positive cell counts in five independent fields under $\times 20$ objective lens is shown with $r$ (Spearman) and P-values indicated.

Representative micrographs of the above-mentioned stromal cells are shown in Additional file 1: Figure S3. Statistics of positive cell counts and their correlation with let-7d expression level in tumor tissue are shown in Additional file 2: Table S2. There were no statistically significant links between let-7d level and counts of cancer associated fibroblasts, Tregs, and mast cells, but an inverse correlation between let-7 $\mathrm{d}$ and macrophage number was observed (Spearman's $r=-0.393, P=0.0003$ ) (Figure 1E). This suggests a regulatory role of let-7d for macrophage infiltration in RCC stroma.

\section{Overexpression of let-7d impedes RCC cell growth and migration of RCC cells in vitro}

The low let-7d level in RCC prompted us to investigate whether let-7d functions as a tumor suppressor in RCC. We infected the RCC cell lines $786 \mathrm{O}$ and 769P with prilet-7d lentivirus and measured its effects on cell proliferation and migration in vitro. After the infection, let-7d levels in $786 \mathrm{O}$ and 769P cells were upregulated (9.6- and 5.3-fold, respectively; Figures $2 \mathrm{~A}$ and $2 \mathrm{~B}$ ), and their proliferation and migration evaluated by proliferation assay (Figures $2 \mathrm{C}$ and $2 \mathrm{D}$ ), Boyden chamber assay (Figures $2 \mathrm{E}$ and $2 \mathrm{H}$ ), and wound-healing assay (Figures $2 \mathrm{~F}, \mathrm{G}$ and $\mathrm{I}$ ) were significantly inhibited, as compared with the cells infected with the control lentivirus. These results indicate that let-7d may be a tumor suppressor in RCC.

\section{Overexpression of let-7d decreases the monocyte recruitment of RCC cells}

To investigate the potential mechanism of the inverse correlation between let-7d expression and tumor macrophage infiltration in RCC, we performed chemotaxis assays with PBMCs in the upper chamber and conditioned medium from let-7d-overexpressing $786 \mathrm{O}$ or $769 \mathrm{P}$ cells or from control cells in the lower chamber. We found that overexpression of let-7d in RCC cells led to decreased migration of PBMCs compared with vehicle control (Figure 2J). These results suggest the regulatory role of let-7d in the inhibition of chemotactic activity leading to the decrease of macrophage infiltration in RCC.

Overexpression of let-7d inhibits the growth, metastasis, and tumor macrophage infiltration of RCC in animal models

We then used the cell-derived xenograft (CDX) model in nude mice to investigate the effects of let-7d overexpression on RCC. The growth of let-7d overexpressing $786 \mathrm{O}$ cells was significantly inhibited and the mean tumor weight was reduced by $66.4 \%$ as compared with the controls (Figures $3 \mathrm{~A}$ and $3 \mathrm{~B})$. The number of metastatic colonies 
Table 1 Relationship between let-7d expression and clinicopathological features in RCC patients

\begin{tabular}{|c|c|c|c|c|}
\hline \multirow[b]{2}{*}{ Variable } & \multirow[b]{2}{*}{ Case no. } & \multicolumn{3}{|c|}{ Let-7d expression ${ }^{1}$ (RQ: $2^{-\Delta C t}$ ) } \\
\hline & & Median & Range & $\mathrm{P}^{2}$ \\
\hline Gender & & & & 0.036 \\
\hline Male & 51 & $5.7 \times 10^{-3}$ & $1.0 \times 10^{-5}-1.06$ & \\
\hline Female & 29 & 0.027 & $3.1 \times 10^{-5}-1.14$ & \\
\hline Age & & & & 0.19 \\
\hline$<60$ (median) & 35 & $7.4 \times 10^{-3}$ & $2.1 \times 10^{-5}-0.655$ & \\
\hline$\geq 60$ & 45 & $8.5 \times 10^{-3}$ & $1.0 \times 10^{-5}-1.14$ & \\
\hline T stage & & & & $<0.0001$ \\
\hline $\mathrm{T} 1$ and $\mathrm{T} 2$ & 50 & 0.046 & $1.9 \times 10^{-4}-1.14$ & \\
\hline T3 & 30 & $9.2 \times 10^{-4}$ & $1.0 \times 10^{-5}-0.067$ & \\
\hline G grade & & & & $<0.0001$ \\
\hline G1 & 27 & 0.28 & $2.8 \times 10^{-3}-1.14$ & \\
\hline $\mathrm{G} 2$ & 38 & $5.8 \times 10^{-3}$ & $2.1 \times 10^{-5}-0.061$ & \\
\hline G3 & 15 & $2 \times 10^{-4}$ & $1.0 \times 10^{-5}-6.8 \times 10^{-3}$ & \\
\hline Histological type & & & & 0.069 \\
\hline papillary RCC & 2 & 0.026 & & \\
\hline chromophobe RCC & 3 & $2 \times 10^{-4}$ & & \\
\hline clear cell RCC & 75 & 0.011 & $1 \times 10^{-5}-1.14$ & \\
\hline Vascular invasion & & & & 0.010 \\
\hline positive & 15 & 0.0014 & $1 \times 10^{-5}-0.18$ & \\
\hline negative & 65 & 0.017 & $2.1 \times 10^{-5}-1.14$ & \\
\hline
\end{tabular}

${ }^{1}$ Let-7d expression was normalized to U6.

${ }^{2}$ Mann-Whitney test for the comparison between two groups or Kruskal-Walis test for more groups.

(Figures 3C and 3D) and the quantification of humanspecific Alu-sequence (Figure 3E) in the mouse lung were also significantly reduced. Tumor macrophages as indicated by $\mathrm{CD}^{2} 8^{+}$cells in let-7d overexpressing xenografts were decreased by $52.7 \%$ as compared with the controls (Figures 3F and 3G).

We further used the patient-derived xenograft model (PDX), a valuable tool for preclinical trials, in NOD/SCID mice to evaluate the effect of let-7d overexpression on RCC growth and metastasis. Early passage (2nd passage) of the RCC xenografts recapitulated the morphologic features of the original clinical tumor (Figure $3 \mathrm{H}$ ). The let-7d level in PDX was similar to that in original human RCC and was significantly decreased as compared with that in normal adjacent tissues (Figure 3I). By intratumoral injection of cholesterol-conjugated let-7d mimics [23], we found that let-7d level in tumors was elevated by 8.6-fold as compared with that in controls (Figure 3I). Consistent with the results in the CDX model, tumor growth was suppressed and tumor weight was decreased after the intratumoral injection of let-7d mimics (Figures 3J and $3 \mathrm{~K}$ ). The number of metastatic colonies and the quantification of human-specific Alu-sequence in mouse lung were also reduced (Figures $3 \mathrm{~L}-\mathrm{N}$ ). The results from both the CDX and PDX animal models suggest that overexpression of let-7d in RCC results in dramatic repression of RCC growth, metastasis, and macrophage infiltration, and that administration of let-7d to tumor tissue may be a therapeutic alternative for RCC.

\section{COL3A1 and CCL7 are direct let-7d target genes in RCC cells}

To investigate the mechanism involved in the suppression effect of let-7d on tumor growth, metastasis, and macrophage infiltration in RCC, we first performed in silico search for the target mRNAs using three algorithms (MiRanda, PicTar, and TargetScan), and obtained a list of predicted target mRNAs of let-7d. The genes potentially involved in tumor growth, metastasis, and chemotaxis activity of RCC were then selected through data mining using the Gene Expression Omnibus Database [24]. Preliminary semi-quantitative RT-PCR screening identified downregulation of COL3A1 and CCL7 mRNA following forced expression of let-7d (data not shown). Quantitative real-time RT-PCR, western blot and ELISA confirmed that the expressions of COL3A1 and CCL7 were significantly decreased at both mRNA and protein levels in 7860 and $769 \mathrm{P}$ cells transfected with pri-let-7d (Figures 4B-D). Immunohistochemistry staining also showed that COL3A1 and CCL7 were decreased in CDX and PDX samples in which let-7d was overexpressed (Figure 4E). 


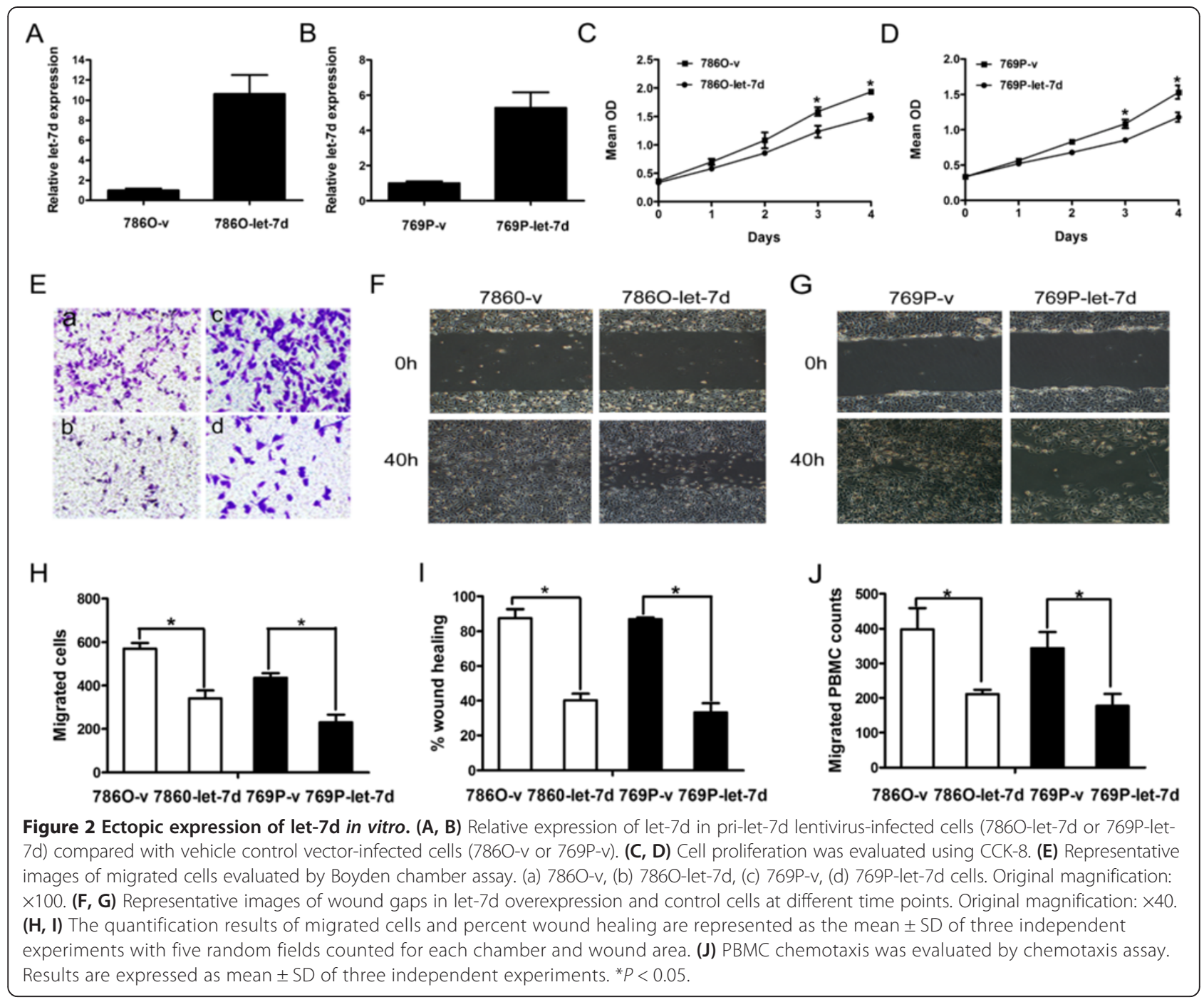

To determine whether the two mRNAs are bona fide targets of let-7d, the 3'-UTRs flanking the putative binding sites of let-7d in COL3A1 and CCL7 mRNAs were cloned into reporter plasmids immediately downstream of luciferase cDNA. The reporter plasmids containing the mutant putative binding sites of let-7d were also constructed as the controls (Figure 4A). Luciferase activities decreased significantly in the cells transfected with the wild-type reporter plasmids but not in those transfected with the mutant plasmids (Figure 4F). These findings indicate that the 3'-UTRs in COL3A1 and CCL7 mRNAs are the target sites, through which let-7d modulates the expressions of COL3A1 and CCL7.

Rescue of COL3A1 and CCL7 overcomes the effects of let-7d To confirm that COL3A1 and CCL7 functions downstream of let-7d, we performed rescue experiments by culturing RCC cells in the presence of purified COL3A1 or CCL7. Addition of $0.2 \mu \mathrm{g} / \mathrm{mL}$ COL3A1 restored the growth and migration of 786O-let-7d and 769P-let-7d cells (Figure $5 \mathrm{~A}-\mathrm{C}$ ). Addition of $10 \mathrm{ng} / \mathrm{mL}$ CCL7 restored the macrophage recruitment of these two cells (Figure 5D). However, addition of COL3A1 did not reverse the inhibitory effects of let-7d overexpression on macrophage recruitment, and CCL7 did not impact growth and migration of RCC cells (data not shown). These data demonstrate that let-7d suppresses RCC cell growth, metastasis, and macrophage recruitment by directly targeting COL3A1 and CCL7.

Inhibition of let-7d in OS-RC-2 cells increased the expression of COL3A1 and CCL7 and enhanced cell proliferation, migration, and monocyte recruitment of the cells

To further assess whether let-7d is involved in the proliferation, migration, and tumor macrophage infiltration of RCC by targeting COL3A1 and CCL7, we inhibited the expression of let-7d with a synthesized let-7d inhibitor in a relatively low metastatic OS-RC-2 cell line with high 


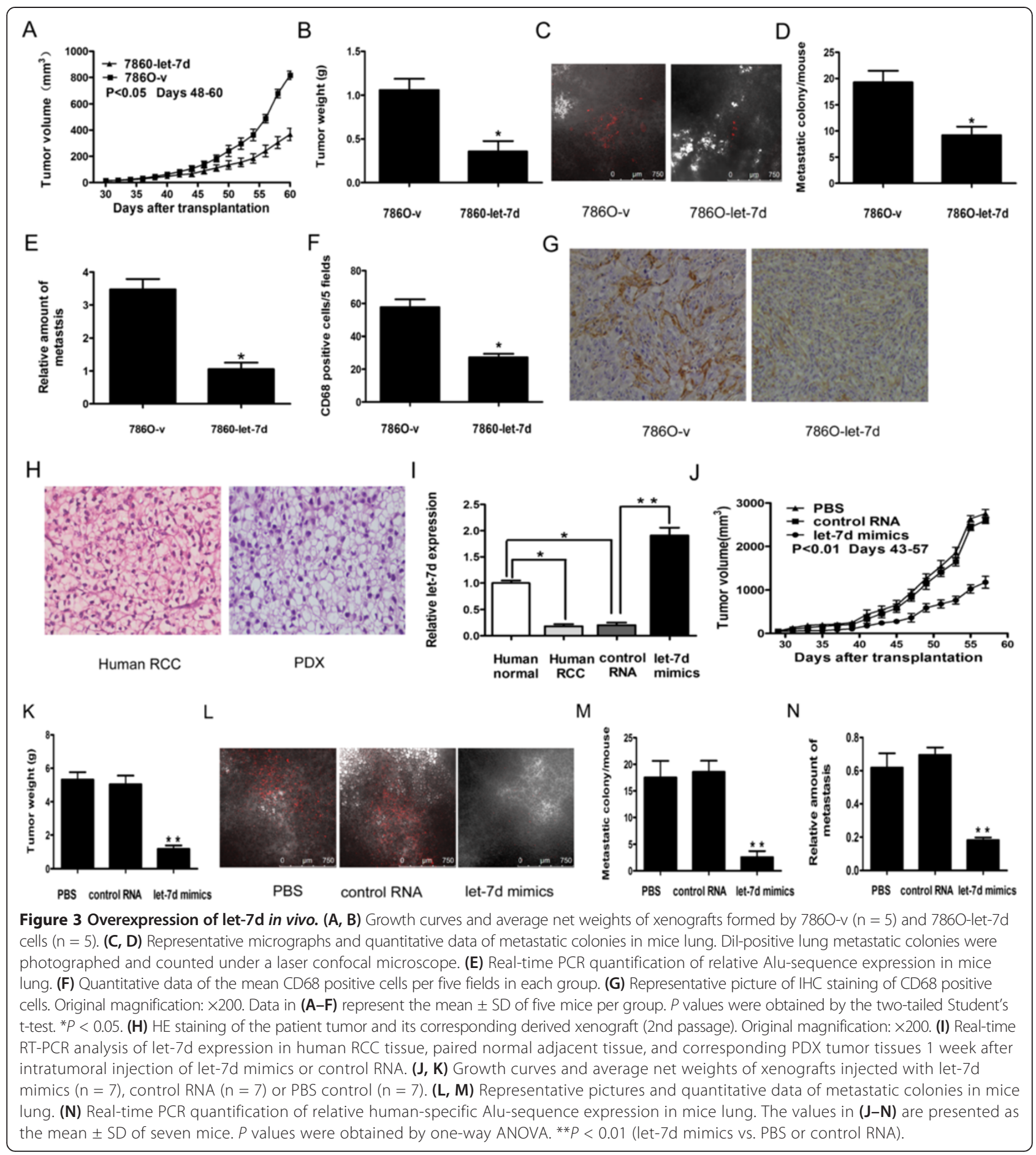

endogenous let-7d expression level (Figure 6A). The levels of endogenous COL3A1 and CCL7 mRNA were increased by 3.1 - and 2.9 -fold, respectively, upon let-7d inhibition compared with the negative control (Figure 6B). Furthermore, western blot and ELISA assay showed that the protein levels of these two genes were upregulated following let-7d repression (Figure 6C, D). The inhibition of let-7d in OS-RC-2 cells significantly increased cell proliferation, migration, and PBMC chemotaxis. Additionally, the increased chemotaxis was eliminated by the addition of $1 \mu \mathrm{g} / \mathrm{mL}$ CCL7 neutralizing antibody in conditioned medium (Figure 6E-J). These data further support the notion that inhibition of let-7d in RCC increases cell proliferation, migration, and macrophage recruitment through the modulation of its targets COL3A1 and CCL7. 


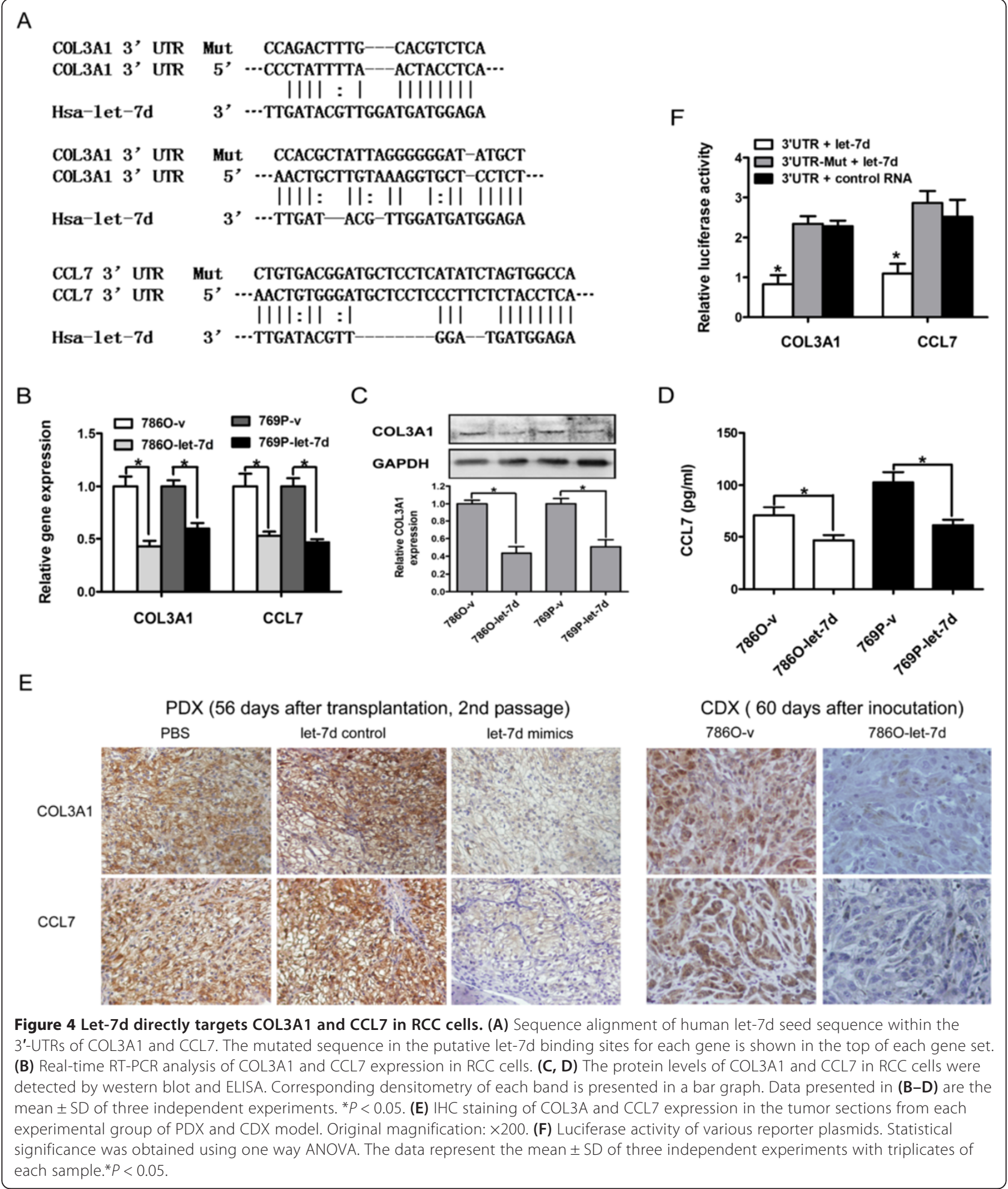

Let-7d expression inversely correlated with COL3A1 and CCL7 mRNA levels in RCC tissues

We analyzed the relationship between level of let-7d and the mRNA levels of COL3A1 and CCL7 in clinical RCC tissues by quantitative real-time RT-PCR Significant inverse correlation was found between let-7d level and COL3A1 mRNA level in the 80 clinical RCC samples (Figures $6 \mathrm{~K}$ and $6 \mathrm{~L}$ ), and between let-7d level and CCL7 mRNA level in the clinical RCC samples with T3 stage. 


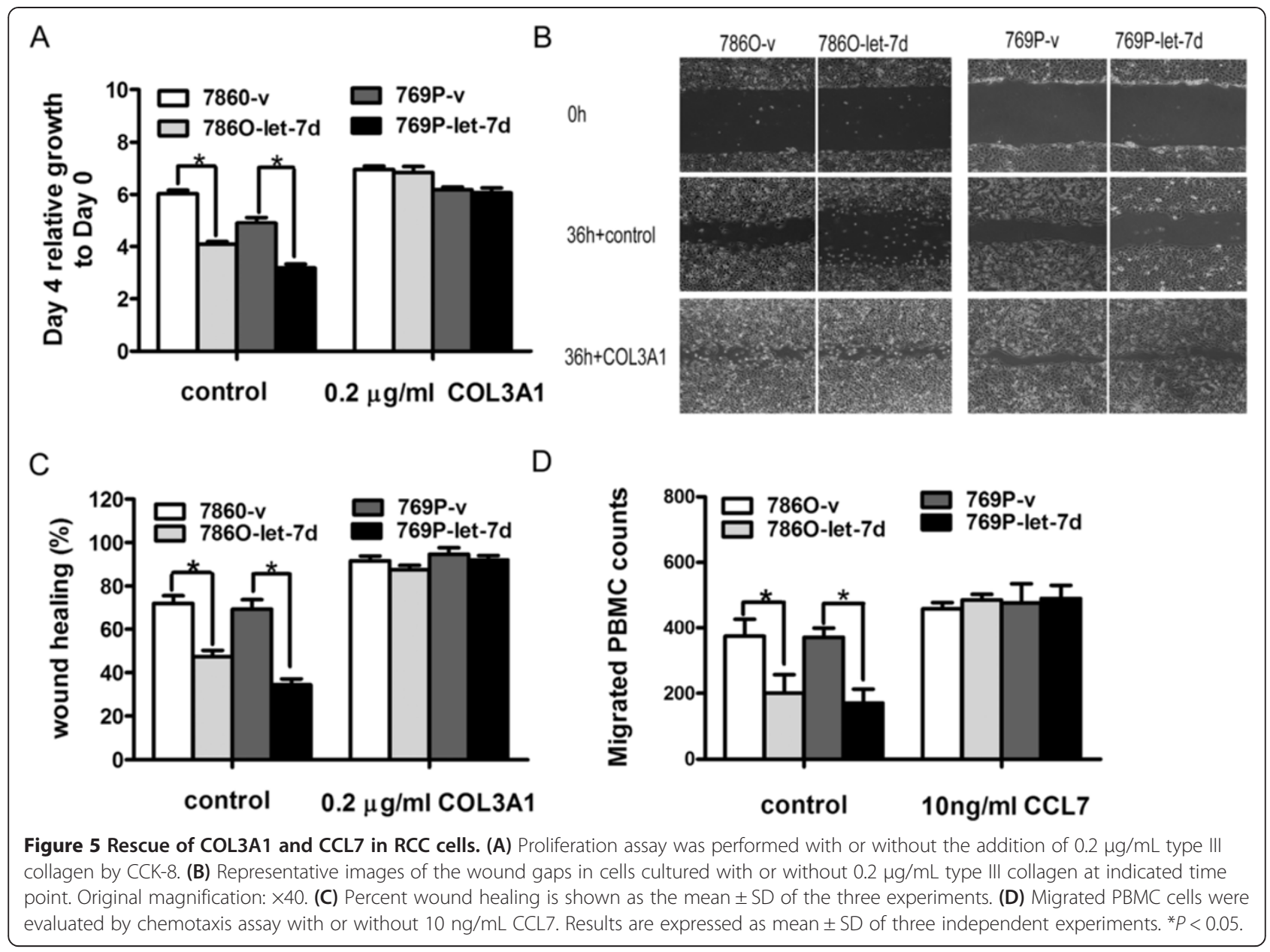

\section{Discussion}

Let-7 $\mathrm{d}$ belongs to the let-7 family that functions as tumor suppressor in many types of cancer [12]. Reported direct targets of let-7 include oncogenes such as RAS, MYC, and HMGA2 [25]. In this study, we demonstrated the tumor suppressive role of let-7d in RCC and validated that the targets of let-7d were COL3A1, an important stroma component, and CCL7, a chemokine attracting monocytes to tumor tissue. Let-7d expression was negatively correlated with COL3A1, CCL7 and CD68 ${ }^{+}$cells in RCC tissues.

Collagen is the most abundant ECM protein in stroma, and contributes to the tumor progression in tumor stroma [10]. Elevated deposition of collagen has been particularly associated with an altered stroma during breast tumorigenesis and correlated with increased breast cancer risk [26]. Lysyl oxidase is an ECM crosslinking enzyme. Collagen crosslinking-mediated matrix stiffening increases integrin clustering, which leads to phosphorylation of focal adhesion kinase and activation of extracellular signalregulated kinase [27]. These changes are all involved in cell migration, invasion and proliferation, leading to tumor progression [28]. COL3A1, also known as collagen, type
III, alpha 1 , is a fibrillar collagen found in extensible connective tissues. The increase of COL3A1 and COL1A1 are found in epithelial ovarian cancers and are prognostic markers of poor prognosis [29]. Interestingly, COL3A1 is the target of miR-29 family, and downregulation of this miRNA family is responsible for the increased invasiveness of lung cancer [30,31]. Here, we validated through in vitro and in vivo research that COL3A1 is a functional direct target gene of let-7d. Importantly, COL3A1 expression was inversely correlated with let-7d levels in RCC clinical specimens. Our findings suggest the involvement of the let-7d-COL3A1 regulatory pathway in RCC growth and metastasis.

Macrophages, which are abundant in the tumor microenvironment, were proven to promote cancer initiation and malignant progression by persuasive clinical and experimental evidence [32]. Tumor-associated macrophages are also involved in tumor progression of RCC and can be used as a potential therapeutic target for metastatic RCC [33]. CCL7 (monocyte chemotactic protein-3, MCP-3) is a member of the $\mathrm{C}-\mathrm{C}$ chemokine subfamily. CCL7 has been found to be overexpressed in gastric cancer tissues and is 


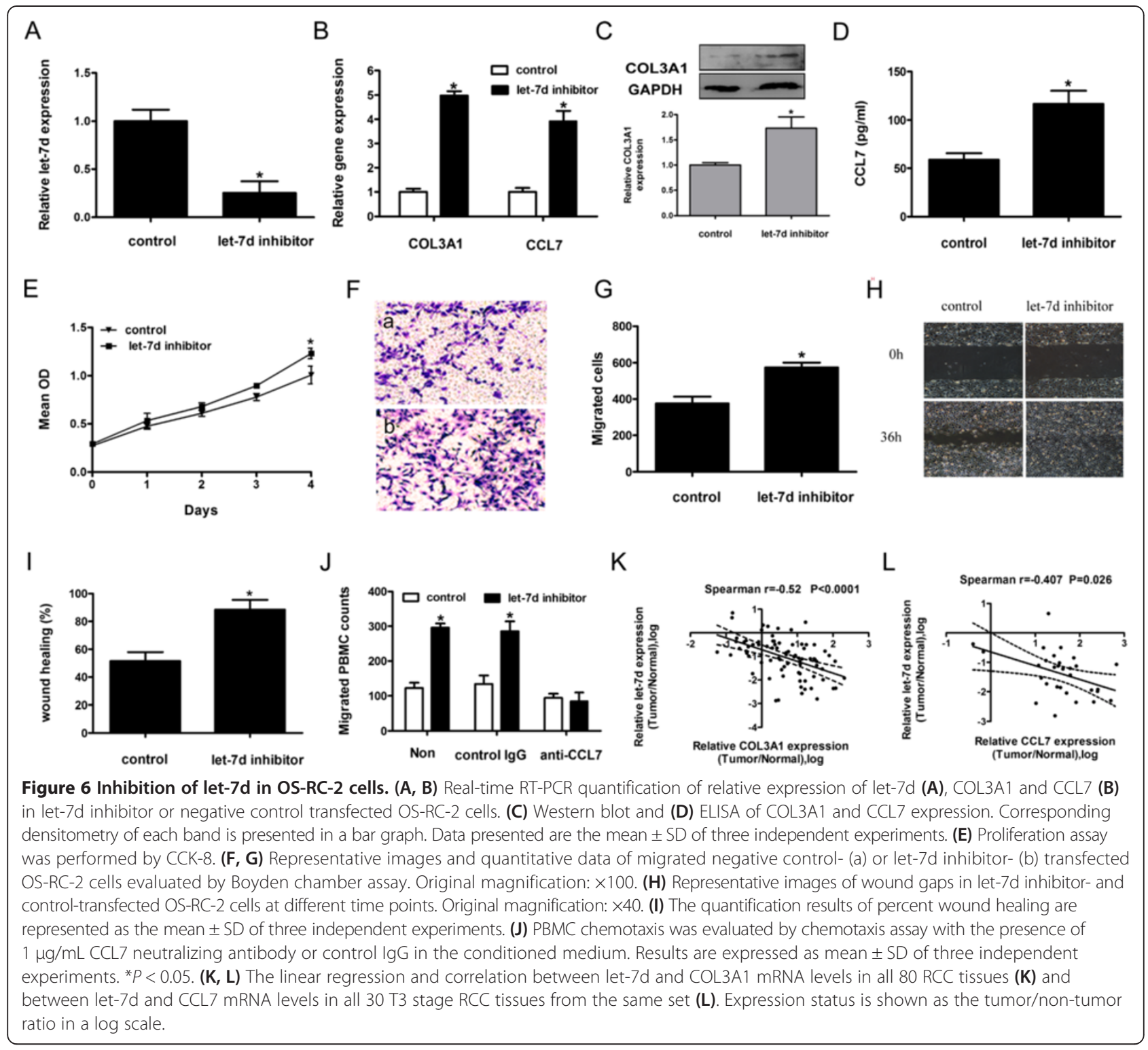

associated with tumor lymph node metastasis and poor prognosis [34]. Furthermore, CCL7 is more abundant in metastatic tumor site than in the primary site, and is associated with macrophage infiltration in tumor [35]. For example, CCL7 is higher in brain metastatic RCC than in primary RCC [36]. CCL2 is another potent macrophage chemoattractant chemokine belonging to the same chemokine family with CCL7. CCL2 recruits macrophages to facilitate metastasis of breast cancer [37]. In RCC [38], however, CCL2 was shown to be of minor importance in the recruitment of macrophages that preserve diverse tumor-promoting functions. CCL7 promotes the invasion and migration of oral squamous cell carcinoma cells through directly binding to its receptor [39]. Interestingly, we found that the suppressed proliferation and migration in let-7d overexpressing RCC cells could not be restored by the addition of exogenous CCL7 alone, probably due to the multiple pathways downstream of let-7d or the lack of functional CCL7 receptor in RCC cells. However, a strong inverse correlation between let-7d expression and number of infiltrated macrophage was found in our clinical RCC samples. Therefore, there is an indirect role of CCL7 in RCC malignancy via the let7d-CCL7-macrophage chain. The negative correlation between let-7d expression and CCL7 in T3 stage RCC tissues additionally highlights the role of CCL7 in tumor invasion and metastasis. CCL7 may be functionally involved in RCC malignant progression and may be used in chemokine target therapy for RCC.

In summary, our results indicate that the tumor suppression role of let-7d in RCC may be partially ascribed to its ability to decrease collagen expression and macrophage 
recruitment through targeting COL3A1 and CCL7 mRNAs. If sufficient let-7d is present in $\mathrm{RCC}$, tumor stroma will be remodeled and cancer cells will be suppressed. It should be noted that let-7d may possess various functions owing to its pleiotropic regulation of genes. It is our expectation that more functional let-7d target genes will be identified in the near future. Further studies are required to fully illustrate their functional roles and interactions with tumor-associated stroma and to determine whether let-7d can be used for the therapy of metastatic RCC, as described in other cancers [23].

\section{Conclusions}

This study demonstrated that let-7d may suppress RCC growth, metastasis and tumor macrophage infiltration at least partially through targeting COL3A1 and CCL7. Our findings suggest let-7d as a promising target for metastatic RCC therapies.

\section{Methods}

\section{Clinical samples and cell lines}

A total of 80 paired RCC tissues and adjacent normal tissues were obtained with informed consent from patients who underwent surgical resection at Peking University First Hospital between 2012 and 2013. The study was approved by Review Board of Peking University First Hospital. All histological subtypes were classified by the Heidelberg classification [40]. Pathological T stage was classified according to the 2010 TNM classification system [41]. Tumor grade was assessed according to Fuhrman nuclear grade [42]. The presence of vascular invasion includes either microvascular invasion, renal vein invasion or inferior vena cava invasion. Immortalized renal proximal tubule epithelial cell line of HK-2 and human RCC cell lines of A498, 769P, 786O, Caki-1 and Caki-2 were purchased from ATCC (MA, USA). Human RCC cell line OS-RC-2 was obtained from the cell bank at Chinese academy of sciences. Transformed human embryonic kidney cells 293FT were purchased from Invitrogen. A498, 769P, 786O, and OS-RC-2 cells were maintained in RPMI 1640 medium, HK-2, Caki-1 and Caki-2 cells in DMEM/F12 (50:50, v/v) medium, and 293FT cells in DMEM. These media were supplemented with $10 \%$ fetal bovine serum (HyClone), and penicillin $(100 \mathrm{U} / \mathrm{mL}) /$ streptomycin $(100 \mu \mathrm{g} / \mathrm{mL})$ (Invitrogen).

\section{Peripheral blood mononuclear cell (PBMC) isolation}

Heparinized venous blood obtained from RCC patients with informed consent was diluted 1:5 with phosphate buffer saline (PBS) and the $40 \mathrm{~mL}$ diluted blood was then underlaid on $10 \mathrm{~mL}$ of Ficoll (Seromed, Berlin, Germany) in $50 \mathrm{~mL}$ plastic tube. After centrifugation at $400 \times g$ for 20 minutes, PBMC were aspirated from the interface, washed with PBS and resuspended to $4 \times 10^{6}$ cells/mL in complete RPMI 1640 medium [43].

\section{In vivo assay}

All mice were maintained in accordance with the $\mathrm{NIH}$ Guidelines for the Care and Use of Laboratory Animals with the approval of Review Board of Peking University First Hospital, Beijing. Patient derived xenograft (PDX) model was established as previously described [23]. RCC surgical samples were gently grinded, labeled with Cell Tracker $^{\mathrm{TM}}$ CM-Dil dye (MoBiTec, Göttingen, Germany) and subcutaneously injected into NOD/SCID mice (Vitalriver, Beijing, China). The xenograft was then harvested, minced into pieces and transplanted into successive mice. For establishment of RCC cell derived xenograft (CDX) model, 5-week old female BALB/C nude mice (Chinese Academy of Sciences, Shanghai, China) were subcutaneously inoculated with 786-O cells labeled with Dil dye and stably transfected with $3 \times 10^{6}$ pri-let-7d or vehicle control lentivirus. Growth of established xenografts was monitored every two days by a caliper for length (L) and width (W) measurement. Tumor volumes were calculated using the formula $\left(\mathrm{L} \times \mathrm{W}^{2}\right) / 2$. In vivo treatment of miRNA mimics in PDX model was performed as previously described [23]. $20 \mathrm{nM}$ chemicallymodified mi-Ribo ${ }^{\mathrm{TM}}$ hsa-let-7d mimics or mi-Ribo ${ }^{\mathrm{TM}}$ hsa-let7d control (Ribobio Co., Guangzhou, China) in $50 \mu \mathrm{L}$ PBS mixed with $50 \mu \mathrm{L}$ in vivo transfection reagent Entranster $^{\text {TM }}$-in vivo (Engreen, Beijing, China) were locally injected into the tumor mass once every 3 days for 3 weeks. Quantification of the RCC cell lung metastatic colonies were obtained by examining the mice lung using the TCS 4D laser scanning confocal microscope (Leica, Heidelberg, Germany).

\section{RNA extraction and real-time RT-PCR}

Total RNA was extracted using miRNeasy Mini Kit (Qiagen, Hilden, Germany). For miRNA quantification, 100 ng total RNA was either reverse transcribed directly using stem-loop primers [44], or was polyadenylated with polyA polymerase (NEB, Beverly, MA, USA) then reverse-transcribed with an oligo-dT adapter primer into cDNAs for quantitative real-time PCR [45]. Although both reverse-transcription methods yield reliable and comparative results, the polyA polymerase tailing method was used in this experiments unless specified, given that it allows measuring multiple target miRNAs with one RT reaction. For mRNA analyses, cDNAs were synthesized from $2 \mu \mathrm{g}$ total RNA, using oligo $(\mathrm{dT})_{15}$ primers and Moloney murine leukemia virus reverse transcriptase (Invitrogen). Quantitative real-time PCR was performed using the SYBR Green PCR Master Mix (Toyobo, Osaka, Japan) in a final volume of $10 \mu \mathrm{L}$ in ABI 7500 Fast PCR machine. The expression of miRNA and mRNAs were normalized to U6 and GAPDH, respectively. Data are presented as relative quantification (RQ) based on the calculation of $2^{-\Delta C t}$. $\Delta C t$ was derived from subtracting 
the $\mathrm{Ct}$ value of reference cDNA from the $\mathrm{Ct}$ value of the cDNA of interest. (For a list of all the primers, see Supporting Data, Additional file 2: Table S1)

\section{Lentiviral transduction}

The human pri-let-7d (primary transcript of let-7d) cDNA sequence was synthesized and inserted into the lentiviral shuttle vector plenti6 (Invitrogen) to generate human plenti6-pri-let-7d plasmid. Plenti6 control and human pri-let-7d lentivirus were generated by transfecting $3 \mu \mathrm{g}$ of plenti6 or plenti6- pri-let-7d and $9 \mu \mathrm{g}$ of ViraPower Packaging Mix (Invitrogen) into 293FT packaging cells using Lipofectamine 2000. After overnight exposure to the transfection mixture, the medium was changed, and the virus-containing supernatant was harvested $48 \mathrm{~h}$ later. The infected cells were selected with $5 \mu \mathrm{g} / \mathrm{mL}$ blasticidin. The antibiotic-resistant clones were pooled and used for subsequent assays.

\section{In vitro cell proliferation, migration, wound healing, and chemotaxis assays}

The cell proliferation assay was performed by using Cell Counting Kit-8 (CCK-8, Dojindo, Kumamoto, Japan). Cell migration was evaluated by Boyden chamber assay and wound healing assay [46]. Monocyte chemotaxis was assayed in 24-well transwell plates (Costar \#3421) with $5 \mu \mathrm{m}$ pore polycarbonate filter membrane. Briefly, $1 \times 10^{7}$ RCC cells were cultured in $5 \mathrm{~mL}$ complete medium for $24 \mathrm{~h}$, the cultured media were collected after centrifugation and used as conditioned media. PBMC were resuspended in $0.1 \%$ BSA-RPMI medium, $4 \times 10^{5}$ cells in $100 \mu \mathrm{L}$ medium were added to the upper chamber of the 24-transwell apparatus, and $800 \mu \mathrm{L}$ conditioned medium were added in the lower chamber. After incubation for $8 \mathrm{~h}$, cells that migrated though the membrane were fixed with $100 \%$ methanol, stained by Giemsa dye, and counted under a microscope. Five high-power fields $(\times 200)$ were randomly selected and manually counted for each well. The experiment was performed in triplicate (3 wells) with three independent tests. Human recombinant COL3A1 (Fitzgerald, Sudbury, MA) $(0.2 \mu \mathrm{g} / \mathrm{mL})$, human recombinant CCL7 (PeproTech, Rocky Hill, NJ) (10 ng/mL), CCL7 neutralizing antibody and normal goat IgG control antibody (R\&D Systems, Minneapolis, MN) $(1 \mu \mathrm{g} / \mathrm{mL})$ were used in these assays.

\section{Elisa}

Cultured media of RCC cells were used for detection of CCL7 by CCL7 ELISA kits (Ray Biotech, Inc). The optical density (OD) at $450 \mathrm{~nm}$ was quantified with a Multiskan microplate spectrophotometer (Thermo LabSystems, Milford, MA).

\section{Quantitative detection of human tumor cell metastasis}

The detection of RCC metastasis in mice lung was performed as described previously [47]. Genomic DNA was extracted from mouse lung tissues using the EasyPure Genomic DNA Kit (Transgen Biotech, Beijing, China). Quantitative real-time PCR was used to measure human Alu-sequences specific for the most conserved region of humans. The primers for Alu-sequences and PCR conditions were used as previously described [47]. The level of human Alu-sequence was normalized to the amount of mouse/human GAPDH genomic DNA sequence amplified by using mouse/human GAPDH primers [48].

\section{Immunohistochemistry (IHC) and toluidine blue staining}

Paraffin embedded tissues were analyzed using immunohistochemical staining [49] with the following primary antibodies: anti-CD68 antibody (DAKO, Carpinteria, CA), anti- $\alpha$-SMA antibody (DAKO, Carpinteria, CA), anti-CCL7 antibody (Gen Way Biotech, San Diego, CA), anti-COL3A1 antibody (Bioss, Beijing, China), anti-FOXP3 antibody (Biolegend, San Diego, CA) and rabbit anti-Mouse CD68 antibody (Bioss, Beijing, China). For quantification of tumor stromal cells within the tumor area, CD68 was used as a pan-macrophage marker, $\alpha$-SMA was used to detect cancer activated fibroblasts adjacent to RCC cells [50], FOXP3 was used as a specific marker for regulatory T cells (Tregs) [22], and mast cells were assessed using the routine toluidine blue staining method [21]. Each tumor section was evaluated by using $20 \times$ objective lens, and five independent areas with the most abundant positive cells were selected, digitally photographed, and manually counted under a microscope. The average positive cell counts for each patient were used for statistical analysis. For quantification of $\mathrm{CD}^{+} 8^{+}$cells in CDX xenografts, four sections from each xenograft were randomly selected and quantified as described above, the average positive cells for each mouse were used for statistical analysis. Results were confirmed by two pathologists in a double-blind analysis.

\section{Western blot analysis}

The lysates were obtained by lysing cells in lysis buffer containing $50 \mathrm{mM}$ Tris, pH7.4, $150 \mathrm{mM} \mathrm{NaCl}, 0.25 \%$ sodium deoxycholate, 1\% NP-40, 0.1\% SDS, $1 \mathrm{mM}$ PMSF, and complete protease inhibitor cocktail (Roche, Mannheim, Germany). Equal amounts of total protein were subjected to $10 \%$ SDS-PAGE and blotted onto PVDF membranes (Pall, Pensacola, FL). Western blotting was performed using rabbit anti-human COL3A1 antibody (Bioss, Beijing, China). The blotting membranes were scanned using GeneSnap acquisition software (Syngene, Cambridge, UK) and band densities were quantified with the GeneTool program (Syngene, Synoptics). GAPDH were used as internal control. 


\section{Dual luciferase activity assay}

The 3'-UTR of human COL3A1 and CCL7 containing the putative binding sites and the mutant binding sites of the mature hsa-let-7d were chemically synthesized and inserted immediately downstream of the luciferase cDNA in the pGL3-control vector (Promega, Madison, WI) by GenePharma (Shanghai, China) to form pGL3-COL3A1, pGL3-CCL7 and pGL3-COL3A1-Mut, pGL3-CCL7-Mut constructs. Twenty-four hours before transfection, $786 \mathrm{O}$ and 293FT cells were plated at $1.5 \times 10^{5}$ cells/well in 24-well plates. $0.5 \mu \mathrm{g}$ of pGL3 constructs plus $0.08 \mu \mathrm{g}$ of pRL-TK (Promega) were transfected in combination with 60 pmol of either a stability-enhanced nontargeting RNA control oligonucleotide or stability-enhanced hsa-let-7d oligonucleotides (GenePharma, Shanghai, China) using Lipofectamine 2000 (Invitrogen). After 48 h, luciferase activity was measured using the Dual Luciferase Reporter Assay System (Promega). Firefly luciferase activity was normalized to renilla luciferase activity for each transfected well. The results were obtained from three independent experiments and each one was performed in triplicate.

\section{Inhibition of let-7d with a miRNA inhibitor}

The chemically-modified mi-Ribo ${ }^{\text {Tw }}$ hsa-let-7d inhibitor or mi-Ribo ${ }^{\text {ma }}$ hsa-let-7d negative control oligonucleotides were synthesized by Ribobio Co. (Guangzhou, China). RCC cells were transfected using Lipofectamine 2000. Cells were collected and assayed at $72 \mathrm{~h}$ post-transfection.

\section{Statistical analysis}

Data are presented as mean \pm SD and were analyzed using the statistical package SPSS17.0 or GraphPad Prism software 5.0. The significance of differences between two groups was determined using a two-sided Student's t-test. In case of multiple tests, one-way ANOVA followed by Bonferroni-Holm procedure was applied. Correlation was performed using two-tailed Spearman's test. $P \leq 0.05$ was considered statistically significant.

\section{Additional files}

Additional file 1: Figure S1. SYBR green real-time RT-PCR analysis shows that there is no significant difference in let-7a expression level between RCC tissue and paired adjacent normal tissue. Horizontal lines represent the relative mean values of let-7a expression for each series of samples. Figure S2. SYBR green real-time RT-PCR analysis demonstrates that there is no significant difference in let-7d expression level in adjacent normal tissues between male and female patients. Horizontal lines represent the relative mean values of let-7d expression for each series of samples. Figure S3. Representative pictures of positive stromal cells (arrow) in RCC. (Original magnification: $\times 200$ ). (a) CD68+ macrophages. (b) a-SMA positive cancer associated fibroblasts. (c) Toluidine blue metachromatic mast cells. (d) FOXP3+ T-regulatory cells.
Additional file 2: Table S1. Primer sequences. Table S2. Statistics of positive cell counts and their correlation with let-7d expression level in tumor tissue.

\section{Abbreviations}

RCC: Renal cell carcinoma; PDX: Patient derived xenograft; CDX: Cell derived xenograft; Dil: 1,1-dioctadecyl-3,3,3',3'-tetramethyl-indocarbocyanine perchlorate; RT-PCR: Reverse transcription-polymerase chain reaction; RQ: Relative quantification; ECM: Extracellular matrix.

\section{Competing interests}

The authors declare that they have no competing interests.

\section{Authors' contributions}

B.Su, B.Shi, Z.Z, Y.X., F.X., Z.G., X.L., W.Z., Z.Q.Z. and L.Z. conceived the experiments and analysed the data; Q. S. and J. W. examined the tumor sections. B.Su, W.Z., B.Shi, Z.Z, Y.X., F.X., X.Z., J.L. and Z.G. carried out experiments; B.Su and Y.X. drafted the manuscript. Z.Q.Z. and L.Z. supervised research project, acted as corresponding authors and did the revisions. All authors were involved in writing the paper and approved the submitted manuscript.

\section{Acknowledgments}

This study was supported by grants from the Beijing Natural Science Foundation (No.7122183) and the National Natural Science Foundation (Grant No.81372746)

\section{Author details}

'Department of Urology, Peking University First Hospital \& the Institute of Urology, Peking University, Beijing 100034, China. ${ }^{2}$ National Urological Cancer Center, Beijing 100034, China. ${ }^{3}$ Department of Cell Biology, Peking University School of Oncology, Beijing Cancer Hospital and Institute, Beijing 100142, China. ${ }^{4}$ Department of Urology, Peking University Shenzhen Hospital, Shenzhen, Guangdong 518036, China. ${ }^{5}$ Department of Urological pathology, Peking University First Hospital \& the Institute of Urology, Peking University, Beijing 100034, China.

Received: 25 April 2014 Accepted: 26 August 2014 Published: 6 September 2014

\section{References}

1. Ramana J: RCDB: Renal Cancer Gene Database. BMC Res Notes 2012, 5:246.

2. Curti BD: Renal cell carcinoma. JAMA 2004, 292:97-100.

3. Vogelzang NJ, Stadler WM: Kidney cancer. Lancet 1998, 352:1691-1696.

4. Rini BI, Campbell SC, Escudier B: Renal cell carcinoma. Lancet 2009, 373:1119-1132.

5. Motzer RJ, Russo P, Nanus DM, Berg WJ: Renal cell carcinoma. Curr Probl Cancer 1997, 21:185-232

6. Flanigan RC, Salmon SE, Blumenstein BA, Bearman SI, Roy V, McGrath PC, Caton JJ, Munshi N, Crawford ED: Nephrectomy followed by interferon alfa- $2 b$ compared with interferon alfa- $2 b$ alone for metastatic renal-cell cancer. N Engl J Med 2001, 345:1655-1659.

7. Croce CM, Calin GA: miRNAs, cancer, and stem cell division. Cell 2005, 122:6-7.

8. Cui SY, Huang JY, Chen YT, Song HZ, Feng B, Huang GC, Wang R, Chen LB, De W: Let-7c governs the acquisition of chemo- or radioresistance and epithelial-to-mesenchymal transition phenotypes in docetaxel-resistant lung adenocarcinoma. Mol Cancer Res 2013, 11:699-713.

9. Joyce JA, Pollard JW: Microenvironmental regulation of metastasis. Nat Rev Cancer 2009, 9:239-252.

10. Hanahan D, Weinberg RA: Hallmarks of cancer: the next generation. Cell 2011, 144:646-674.

11. Soon P, Kiaris H: MicroRNAs in the tumour microenvironment: big role for small players. Endocr Relat Cancer 2013, 20:R257-R267.

12. Boyerinas B, Park SM, Hau A, Murmann AE, Peter ME: The role of let-7 in cell differentiation and cancer. Endocr Relat Cancer 2010, 17:F19-F36.

13. Bussing I, Slack FJ, Grosshans H: let-7 microRNAs in development, stem cells and cancer. Trends Mol Med 2008, 14:400-409.

14. Thomson JM, Parker J, Perou CM, Hammond SM: A custom microarray platform for analysis of microRNA gene expression. Nat Methods 2004 $1: 47-53$ 
15. Shimizu S, Takehara T, Hikita H, Kodama T, Miyagi T, Hosui A, Tatsumi T, Ishida H, Noda T, Nagano H, Doki Y, Mori M, Hayashi N: The let-7 family of microRNAs inhibits $\mathrm{BCl}-\mathrm{xL}$ expression and potentiates sorafenib-induced apoptosis in human hepatocellular carcinoma. J Hepatol 2010, 52:698-704

16. Zhao B, Han H, Chen J, Zhang Z, Li S, Fang F, Zheng Q, Ma Y, Zhang J, Wu $N$, Yang $Y$ : MicroRNA let-7c inhibits migration and invasion of human non-small cell lung cancer by targeting ITGB3 and MAP4K3. Cancer Lett 2014, 342:43-51.

17. Yu F, Yao H, Zhu P, Zhang X, Pan Q, Gong C, Huang Y, Hu X, Su F, Lieberman J, Song E: let-7 regulates self renewal and tumorigenicity of breast cancer cells. Cell 2007, 131:1109-1123.

18. Shell S, Park SM, Radjabi AR, Schickel R, Kistner EO, Jewell DA, Feig C, Lengyel E, Peter ME: Let-7 expression defines two differentiation stages of cancer. Proc Natl Acad Sci U S A 2007, 104:11400-11405.

19. Johnson SM, Grosshans H, Shingara J, Byrom M, Jarvis R, Cheng A, Labourier E, Reinert KL, Brown D, Slack FJ: RAS is regulated by the let-7 microRNA family. Cell 2005, 120:635-647.

20. Liu Y, Yin B, Zhang C, Zhou L, Fan J: Hsa-let-7a functions as a tumor suppressor in renal cell carcinoma cell lines by targeting c-myc. Biochem Biophys Res Commun 2012, 417:371-375.

21. Tuna B, Yorukoglu K, Unlu M, Mungan MU, Kirkali Z: Association of mast cells with microvessel density in renal cell carcinomas. Eur Urol 2006, 50:530-534.

22. Liotta F, Gacci M, Frosali F, Querci V, Vittori G, Lapini A, Santarlasci V, Serni S, Cosmi L, Maggi L, Angeli R, Mazzinghi B, Romagnani P, Maggi E, Carini M, Romagnani S, Annunziato F: Frequency of regulatory T cells in peripheral blood and in tumour-infiltrating lymphocytes correlates with poor prognosis in renal cell carcinoma. BJU Int 2011, 107:1500-1506.

23. Hou J, Lin L, Zhou W, Wang Z, Ding G, Dong Q, Qin L, Wu X, Zheng Y, Yang Y, Tian W, Zhang Q, Wang C, Zhang Q, Zhuang SM, Zheng L, Liang A, Tao $W$, Cao X: Identification of miRNomes in human liver and hepatocellular carcinoma reveals miR-199a/b-3p as therapeutic target for hepatocellular carcinoma. Cancer Cell 2011, 19:232-243.

24. Gene Expression Omnibus Database. [http://www.ncbi.n/m.nih.gov/geoprofiles/]

25. Roush S, Slack FJ: The let-7 family of microRNAs. Trends Cell Biol 2008, 18:505-516.

26. Provenzano PP, Eliceiri KW, Campbell JM, Inman DR, White JG, Keely PJ: Collagen reorganization at the tumor-stromal interface facilitates local invasion. BMC Med 2006, 4:38.

27. Ng MR, Brugge JS: A stiff blow from the stroma: collagen crosslinking drives tumor progression. Cancer Cell 2009, 16:455-457.

28. Provenzano PP, Inman DR, Eliceiri KW, Keely PJ: Matrix density-induced mechanoregulation of breast cell phenotype, signaling and gene expression through a FAK-ERK linkage. Oncogene 2009, 28:4326-4343.

29. Santala M, Simojoki M, Risteli J, Risteli L, Kauppila A: Type I and III collagen metabolites as predictors of clinical outcome in epithelial ovarian cancer. Clin Cancer Res 1999, 5:4091-4096.

30. Maurer B, Stanczyk J, Jungel A, Akhmetshina A, Trenkmann M, Brock M, Kowal-Bielecka O, Gay RE, Michel BA, Distler JH, Gay S, Distler O: MicroRNA-29, a key regulator of collagen expression in systemic sclerosis. Arthritis Rheum 2010, 62:1733-1743.

31. Rothschild SI, Tschan MP, Federzoni EA, Jaggi R, Fey MF, Gugger M, Gautschi O: MicroRNA-29b is involved in the Src-ID1 signaling pathway and is dysregulated in human lung adenocarcinoma. Oncogene 2012, 31:4221-4232

32. Qian BZ, Pollard JW: Macrophage diversity enhances tumor progression and metastasis. Cell 2010, 141:39-51.

33. Santoni M, Massari F, Amantini C, Nabissi M, Maines F, Burattini L, Berardi R, Santoni G, Montironi R, Tortora G, Cascinu S: Emerging role of tumor-associated macrophages as therapeutic targets in patients with metastatic renal cell carcinoma. Cancer Immunol Immunother 2013, 62:1757-1768.

34. Hwang TL, Lee LY, Wang CC, Liang Y, Huang SF, Wu CM: CCL7 and CCL21 overexpression in gastric cancer is associated with lymph node metastasis and poor prognosis. World J Gastroenterol 2012, 18:1249-1256.

35. Okada M, Saio M, Kito Y, Ohe N, Yano H, Yoshimura S, Iwama T, Takami T: Tumor-associated macrophage/microglia infiltration in human gliomas is correlated with MCP-3, but not MCP-1. Int J Oncol 2009, 34:1621-1627.

36. Wyler L, Napoli CU, Ingold B, Sulser T, Heikenwalder M, Schraml P, Moch H: Brain metastasis in renal cancer patients: metastatic pattern, tumour-associated macrophages and chemokine/chemoreceptor expression. Br J Cancer 2014, 110:686-694.
37. Qian BZ, Li J, Zhang H, Kitamura T, Zhang J, Campion LR, Kaiser EA, Snyder LA, Pollard JW: CCL2 recruits inflammatory monocytes to facilitate breast-tumour metastasis. Nature 2011, 475:222-225.

38. Hemmerlein B, Johanns U, Kugler A, Reffelmann M, Radzun HJ: Quantification and in situ localization of MCP-1 mRNA and its relation to the immune response of renal cell carcinoma. Cytokine 2001, 13:227-233.

39. Jung DW, Che ZM, Kim J, Kim K, Kim KY, Williams D, Kim J: Tumor-stromal crosstalk in invasion of oral squamous cell carcinoma: a pivotal role of CCL7. Int J Cancer 2010, 127:332-344.

40. Kovacs G, Akhtar M, Beckwith BJ, Bugert P, Cooper CS, Delahunt B, Eble JN Fleming $S$, Ljungberg B, Medeiros LJ, Moch H, Reuter VE, Ritz E, Roos G, Schmidt D, Srigley JR, Störkel S, van den Berg E, Zbar B: The Heidelberg classification of renal cell tumours. J Pathol 1997, 183:131-133.

41. Pichler M, Hutterer GC, Chromecki TF, Jesche J, Kampel-Kettner K, Groselj-Strele A, Hoefler G, Pummer K, Zigeuner R: Comparison of the 2002 and TNM classification systems regarding outcome prediction in clear cell and papillary renal cell carcinoma. Histopathology 2010, 2013(62):237-246.

42. Fuhrman SA, Lasky LC, Limas C: Prognostic significance of morphologic parameters in renal cell carcinoma. Am J Surg Pathol 1982, 6:655-663.

43. Sica A, Saccani A, Bottazzi B, Bernasconi S, Allavena P, Gaetano B, Fei F, LaRosa G, Scotton C, Balkwill F, Mantovani A: Defective expression of the monocyte chemotactic protein-1 receptor CCR2 in macrophages associated with human ovarian carcinoma. J Immunol 2000, 164:733-738.

44. Varkonyi-Gasic E, Hellens RP: Quantitative stem-loop RT-PCR for detection of microRNAs. Methods Mol Biol 2011, 744:145-157.

45. Chen C, Ridzon DA, Broomer AJ, Zhou Z, Lee DH, Nguyen JT, Barbisin M, Xu NL, Mahuvakar VR, Andersen MR, Lao KQ, Livak KJ, Guegler KJ: Real-time quantification of microRNAs by stem-loop RT-PCR. Nucleic Acids Res 2005 , 33:e179.

46. Lan L, Han H, Zuo H, Chen Z, Du Y, Zhao W, Gu J, Zhang Z: Upregulation of myosin Va by Snail is involved in cancer cell migration and metastasis. Int J Cancer 2010, 126:53-64.

47. Zijlstra A, Mellor R, Panzarella G, Aimes RT, Hooper JD, Marchenko ND, Quigley JP: A quantitative analysis of rate-limiting steps in the metastatic cascade using human-specific real-time polymerase chain reaction. Cancer Res 2002, 62:7083-7092.

48. Lee RH, Pulin AA, Seo MJ, Kota DJ, Ylostalo J, Larson BL, Semprun-Prieto L Delafontaine P, Prockop DJ: Intravenous hMSCs improve myocardial infarction in mice because cells embolized in lung are activated to secrete the anti-inflammatory protein TSG-6. Cell Stem Cell 2009, 5:54-63.

49. He L, Ding H, Wang JH, Zhou Y, Li L, Yu YH, Huang L, Jia WH, Zeng M, Yun JP, Luo RZ, Zheng M: Overexpression of karyopherin 2 in human ovarian malignant germ cell tumor correlates with poor prognosis. PLOS One 2012, 7:e42992

50. Yao Q, Cao S, Li C, Mengesha A, Kong B, Wei M: Micro-RNA-21 regulates TGF-beta-induced myofibroblast differentiation by targeting PDCD4 in tumor-stroma interaction. Int J Cancer 2011, 128:1783-1792.

doi:10.1186/1476-4598-13-206

Cite this article as: Su et al.: Let-7d suppresses growth, metastasis, and tumor macrophage infiltration in renal cell carcinoma by targeting COL3A1 and CCL7. Molecular Cancer 2014 13:206.

\section{Submit your next manuscript to BioMed Central and take full advantage of:}

- Convenient online submission

- Thorough peer review

- No space constraints or color figure charges

- Immediate publication on acceptance

- Inclusion in PubMed, CAS, Scopus and Google Scholar

- Research which is freely available for redistribution 